Proceedings

\title{
A Novel Approach for a Faster Prototyping of Winter Sport Equipment Using Digital Image Correlation and 3D Printing ${ }^{\dagger}$
}

\author{
Martino Colonna 1,*, Benno Zingerle 1, Maria Federica Parisi ${ }^{~}$, Claudio Gioia ${ }^{1}$, \\ Alessandro Speranzoni ${ }^{1}$, Gregorio Pisaneschi ${ }^{2}$ and Stefano Prosdocimo ${ }^{3}$ \\ 1 DICAM, University of Bologna, 40131 Bologna, Italy; benno.zingerle@studio.unibo.it (B.Z.); \\ mariafederica.paris2@unibo.it (M.F.P.); claudio.gioia2@unibo.it (C.G.); alle.speranzoni@gmail.com (A.S.) \\ 2 DIN, University of Bologna, 40131 Bologna, Italy; gregorio.pisaneschi@unibo.it \\ 3 Dalbello srl, 31011 Casella d'Asolo (TV), Italy; Stefano.Prosdocimo@dalbello.it \\ * Correspondence: martino.colonna@unibo.it; Tel.: +39-339-343-1512 \\ + Presented at the 13th conference of the International Sports Engineering Association, Online, \\ 22-26 June 2020.
}

Published: 15 June 2020

\begin{abstract}
The optimization of sport equipment parts requires considerable time and high costs due to the high complexity of the development process. For this reason, we have developed a novel approach to decrease the cost and time for the optimization of the design, which consists of producing a first prototype by 3D printing, applying the forces that normally acts during the sport activity using a test bench, and then measuring the local deformations using 3D digital image correlation (DIC). The design parameters are then modified by topological optimization and then DIC is performed again on the new 3D-printed modified part. The DIC analysis of 3D-printed parts has shown a good agreement with that of the injection-molded ones. The deformation measured with DIC are also well correlated with those provided by finite element method (FEM) analysis, and therefore DIC analysis proves to be a powerful tool to validate FEM models.
\end{abstract}

Keywords: sport equipment; prototyping; 3D printing; digital image correlation; mechanical properties; winter sports

\section{Introduction}

The optimization of the design of sport equipment made from thermoplastic materials is a difficult task that requires a considerable amount of time and high costs since it is performed with a trial and error approach that necessitates the modification of molds used to produce the parts. This approach increases the time to market of a new product and significantly affect the final costs. This aspect has become even more crucial in the last few years due to the ever-growing request from the market to reduce the weight of sport equipment [1]. An efficient reduction of plastic thickness is very challenging due to the intrinsic difficulty to determine the zones in which the maximum stress is applied. Finite element method (FEM) analysis can be used to predict the deformation and avoid eventual breakages. However, in FEM analysis, it is necessary to know the loads that are applied during the use of the part and this is very difficult to be determined directly. Test benches that measure the flexural stiffness of the parts have been used to determine the flexural behavior of winter sport equipment made of rigid plastic parts [2]. These test benches permit the application of forces that are similar to those applied in real use and are widely employed for lab testing to predict the performances, for example, of ski boots $[1,2]$. However, the sport equipment is generally constituted of several parts made from different materials and thicknesses, and for this reason, it is very difficult to correlate the overall flexural behavior with that of the single parts. Digital image correlation (DIC) 
allows for the measurement of local deformations on a part on which forces are applied. DIC is a technique based on 3D image-tracking of a stochastic pattern of small dots created on the surface that allows one to measure local deformations on 3-axis.

$3 \mathrm{D}$ printing is widely used in R\&D since it allows one to obtain prototypes with a significant time reduction and cost saving when compared with traditional processes [3]. For example, 3D printing has been used to develop and produce snowboard bindings $[4,5]$.

The aim of the present work is to use DIC and 3D printing to perform a more efficient process for the optimization of the design of sport equipment. In particular, we developed a new prototype of a snowboard binding baseplate that was designed using FEM analysis and topological optimization, taking into account the loads applied that were indirectly determined by analyzing the deformation measured using DIC. A comparison of the deformation patterns of 3D-printed and injection-molded parts was also been conducted.

\section{Materials and Methods}

A snowboard binding model "Falcor", manufactured by Union Bindings (Figure 1), was used as a reference binding. The baseplate of the binding is produced by injection molding using Akulon K224 (Nylon 6 with 30\% of glass fibers). A test bench was built to measure the flexural behavior of the binding. The binding was attached with screws to a metallic base and a boot containing a prosthesis was inserted in the binding. The top part of the prosthesis was connected with a metallic cable to a dynamometer with a $500 \mathrm{~N}$ load cell. A speed of $200 \mathrm{~mm} / \mathrm{min}$ was used in the tests and the test was stopped when a $250 \mathrm{~N}$ force was reached both in plantarflexion and in dorsiflexion.

Bindings and test specimens were manufactured by 3D printing using an HP4200 MJF using HP $3 \mathrm{D}$ high reusability polyamide 12 as a base material.
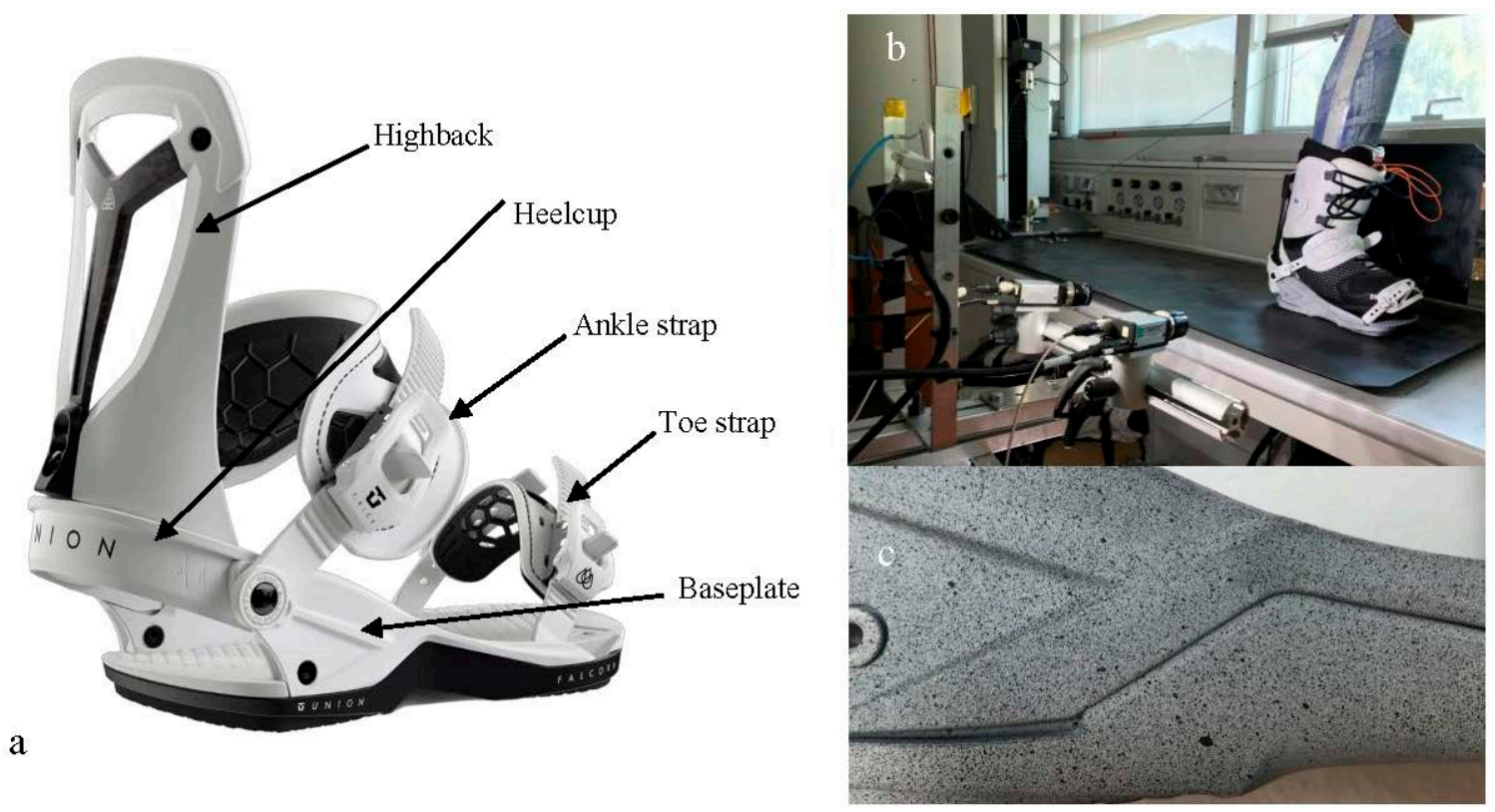

Figure 1. Falcor snowboard binding (a), flexural test bench used and digital image correlation (DIC) equipment (b) and stochastic pattern used to perform DIC analysis (c).

DIC measurements were performed with a Dantec Dynamics Q-400 3D with Xenoplan 1.4/17 Schneider Kreuznach optics using Istra4D Software. The sample was first sprayed with a white color base and then with a black color to create the stochastic pattern on the surface. The values of the strains measured by DIC of different bindings were compared by taking into account the values of the spots in which maximum strain was observed. FEM analysis and topological optimization were conducted using the software Fusion 360 from Autodesk. The boundary conditions were partially determined by analyzing the deformation of the straps using DIC and partially with a trial and error 
approach to minimize the differences between DIC and FEM results. In particular, in plantarflexion, a force of $700 \mathrm{~N}$ applied by the ankle strap, a force of $150 \mathrm{~N}$ induced by the toe strap and a remote force of $700 \mathrm{~N}$ that simulate the effect of the force applied by the high back on the heel cup were used; while in dorsiflexion, a force of $400 \mathrm{~N}$ from the ankle strap and of $100 \mathrm{~N}$ from the toe strap were used in the FEM model.

\section{Results and Discussion}

The analysis of the binding was concentrated on its lower part, known as the baseplate. This part is the more rigid one and its purpose is to transmit the forces from the rider to the snowboard.

Flexural tests were performed to determine the forces that are applied during dorsiflexion and plantarflexion, using a system that has been previously used to measure the flexural stiffness of ski boots [1]. According to the literature [2] all the test parameters were kept constant (temperature, buckles closure, type of prosthesis, movement speed, etc.) during all tests.

Multi jet fusion was the chosen 3D-printing technique since it permits the use of polyamidesthe materials employed in the traditional injection-molding process. Moreover, this technique is known for the very low anisotropy of the printed parts. The choice of polyamides is related also to the fact that they have good impact resistance at low temperature and have been used in a previous scientific work on 3D printing of snowboard bindings [3]. However, the polyamide used for 3D printing possesses a significantly lower elastic modulus (0.90 GPa vs. $5.50 \mathrm{GPa}$ ) compared to that used for the injection molding of the commercial Falcor baseplate used as a reference, which contains $30 \%$ of glass fibers. This difference is responsible for the different deformation curves of the two bindings, as shown in Figure 2. The value of strain measured by DIC in the point of maximum horizontal deformation $(0.0127$ for the 3D-printed one versus 0.0077 for the injection-molded one during dorsiflexion, reported in Figure 3) is not linearly correlated with the modulus difference between the 3D-printed material and the injection-molded one. The reason can be ascribed to the fact that the values of modulus for the injection-molded material are measured on a specimen in which the fibers are all aligned, and this is not generally the case in an object with a complex shape like the snowboard bindings that we are analyzing. Nevertheless, the comparison of the DIC analysis performed during the flexural tests show a very similar pattern of deformation for the injectionmolded and 3D-printed parts (as an example, horizontal deformation in dorsiflexion is shown in Figure 3) indicating that the measure of deformation by DIC of 3D-printed parts can give important information that can be used to predict the behavior of injection-molded parts.

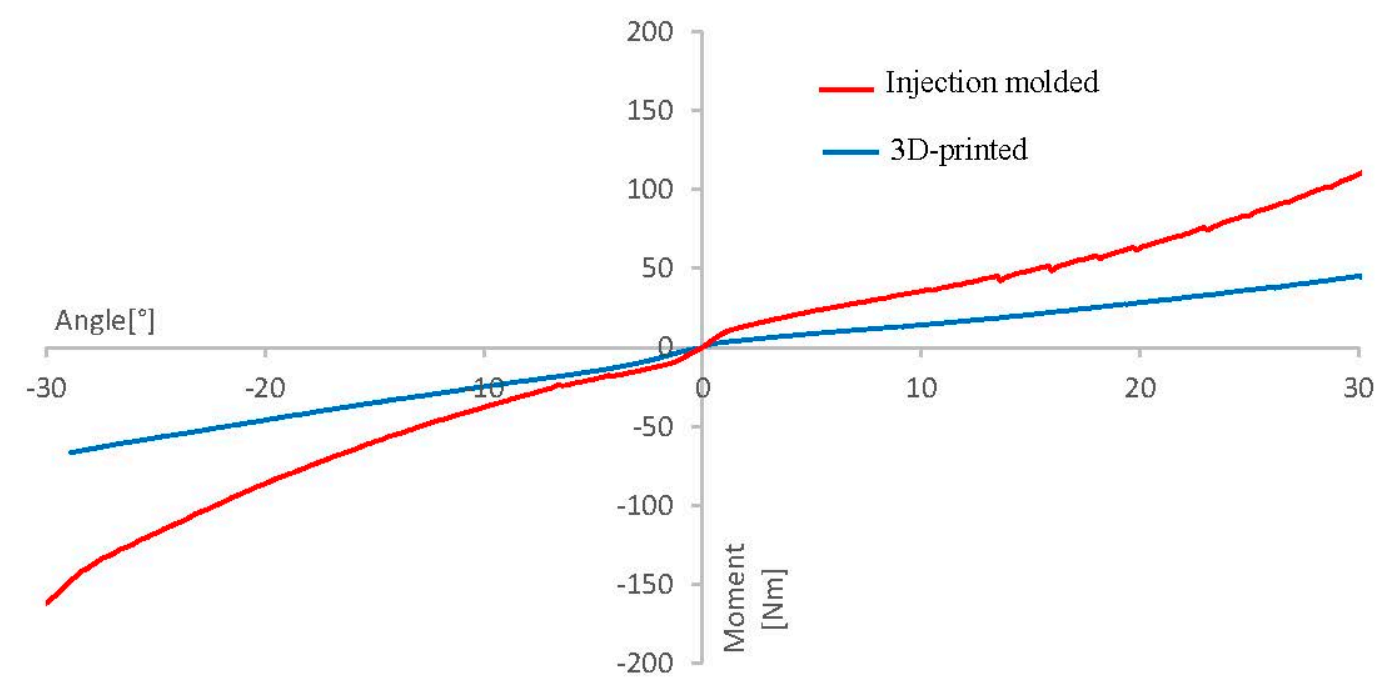

Figure 2. Comparison of dorsiflexion and plantarflexion flex curves of Falcor binding made by injection molding and $3 \mathrm{D}$ printing. 
The FEM analysis was conducted on the basis of the results of DIC and of the forces applied in the test bench as reported in the method section. The comparison of the FEM analysis (Figure 4) with DIC results (Figure 3) show a good agreement on the deformation patterns. Moreover, the values of the strain measured in the zone of the maximum horizontal deformation in dorsiflexion for the FEM analysis are very similar to those observed by DIC (0.0078 vs. 0.0077$)$. Similar results are obtained considering other deformation axis also in plantarflexion. These facts indicate that DIC is a powerful tool to validate FEM simulations.
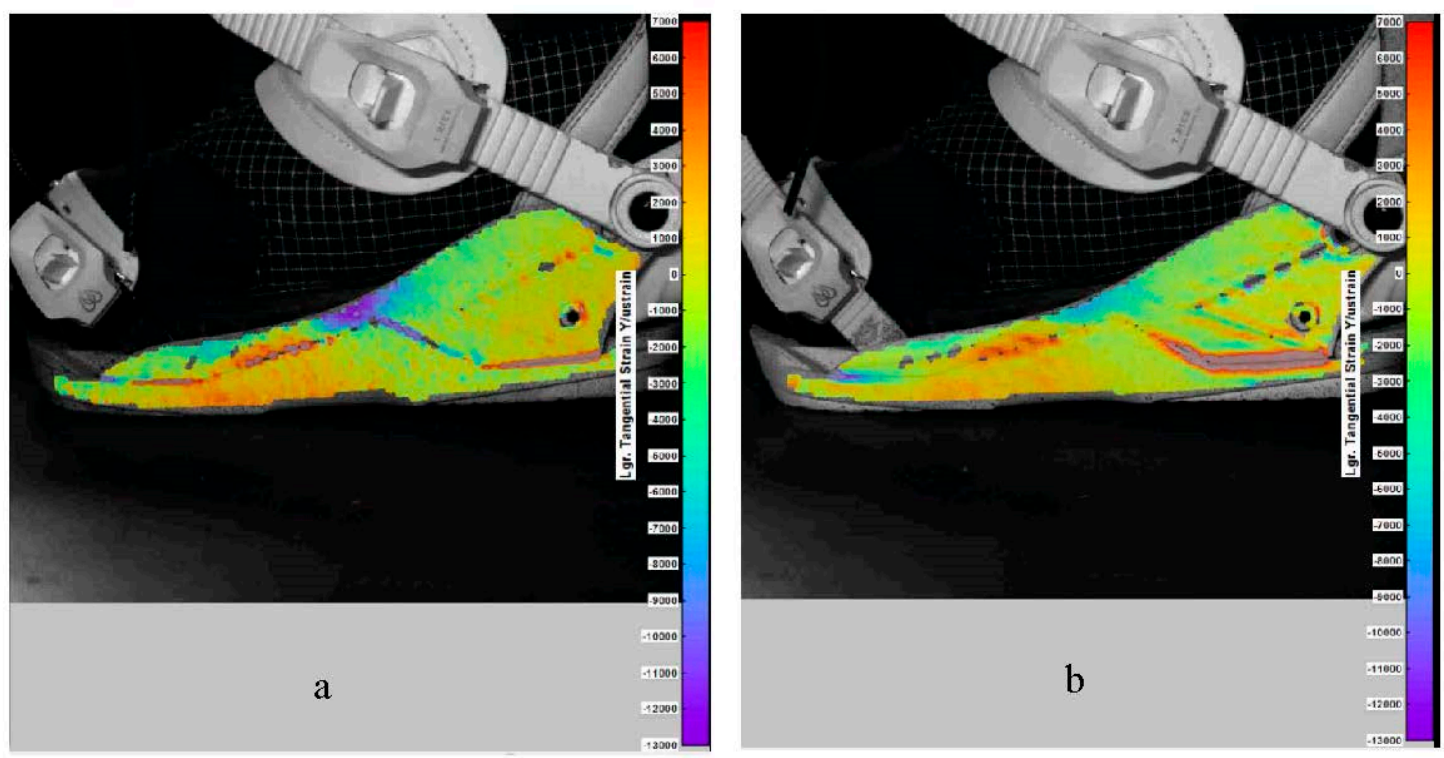

Figure 3. Comparison of deformations (horizontal axis) of a commercial baseplate made by injection molding (b) and a baseplate 3D printed (a) at $250 \mathrm{~N}$, applied on the upper part of the prosthesis in dorsiflexion.

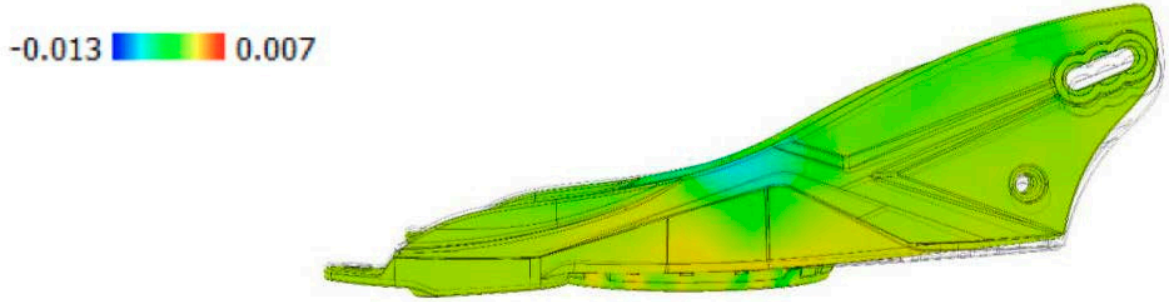

Figure 4. Finite element method (FEM) simulation in dorsiflexion (horizontal axis deformation).

Based on the results of the FEM analysis, a topological optimization was conducted using Autodesk Fusion 360 software. The inputs and constraints given by the topological optimization (Figure 5) were used to complete the design of a new prototype with a reduced weight. FEM analysis showed that the prototype has improved mechanical properties when compared with the commercial baseplate (Figure 6, for example), as shown in the deformation in the plantarflexion. 


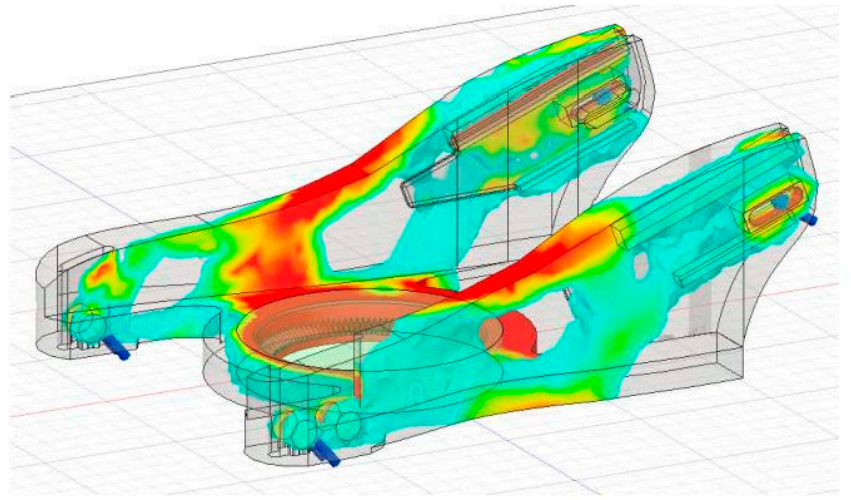

Figure 5. Autodesk Fusion 360 output for the topological optimization of the binding.
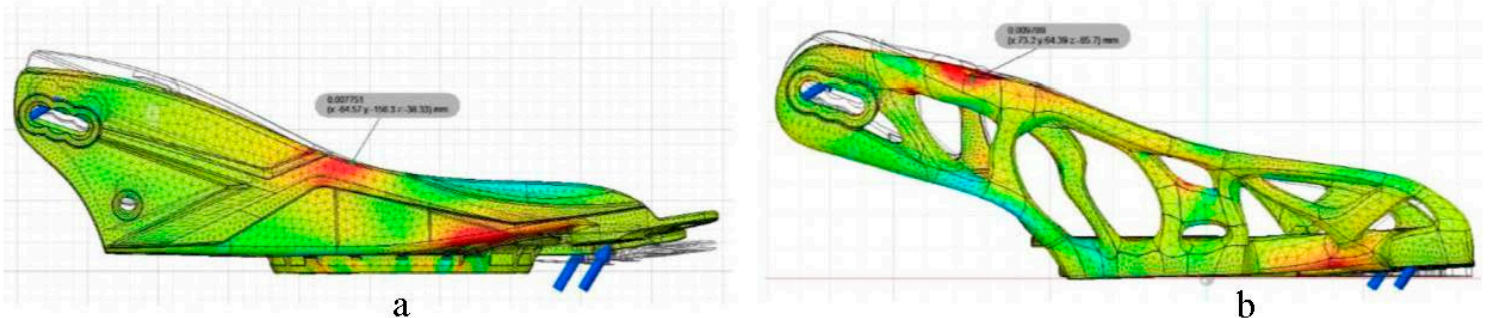

Figure 6. FEM simulation of the deformation in plantarflexion (horizontal axis) of the commercial binding (a) and of the prototype (b).

The prototype baseplate was then produced by 3D printing (Figure 7), showing a $29 \%$ reduction of weight when compared to the commercial baseplate, which was also made by $3 \mathrm{D}$ printing.

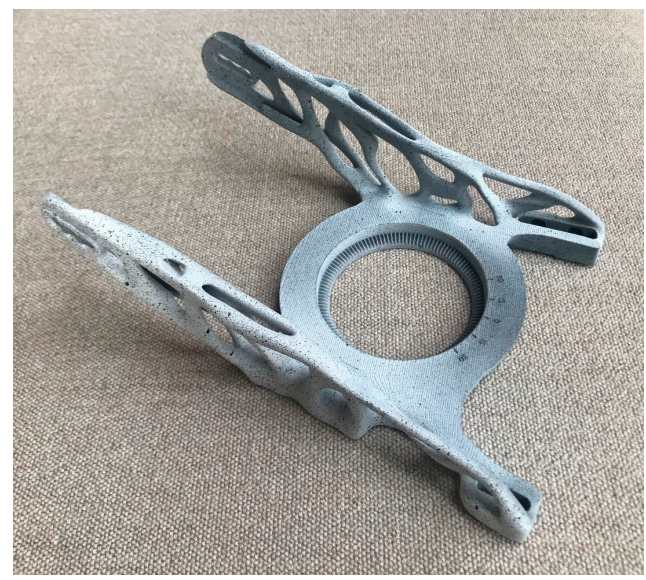

Figure 7.3D-printed prototype.

The 3D-printed baseplate was assembled with the same heel cup, high back and straps of the commercial model and tested in the test bench to measure the flexural stiffness and the deformations caused by DIC.

The results reported in Figure 8 show that the prototype binding has a higher flexural stiffness both in dorsiflexion and plantarflexion when compared to the commercial one, despite its weight being significantly lower. Moreover, the comparison of the DIC analysis (as an example, Figure 9 shows the deformation of the horizontal axis in plantarflexion) shows less pronounced deformation and less localized stress on the prototype. Again, a good agreement can be observed comparing DIC (Figure 6) and FEM analysis deformation patterns (Figure 9). 


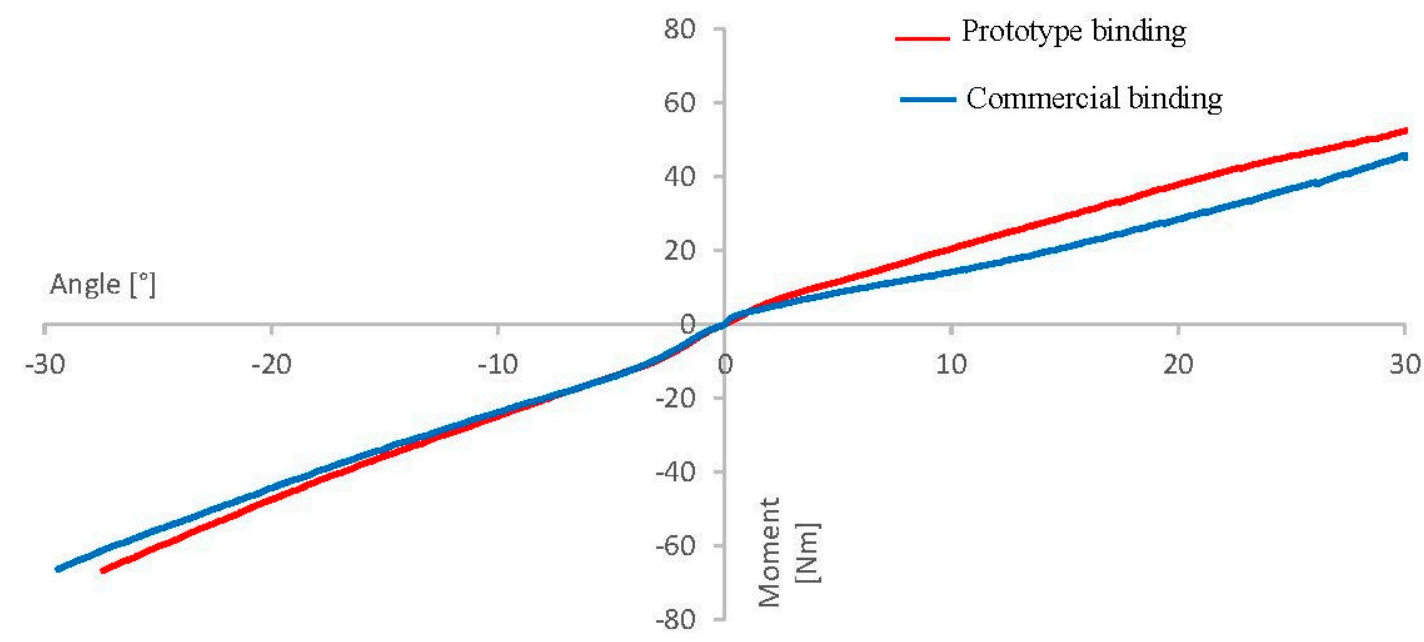

Figure 8. Comparison of the flexural behavior of the prototype and of the commercial binding.
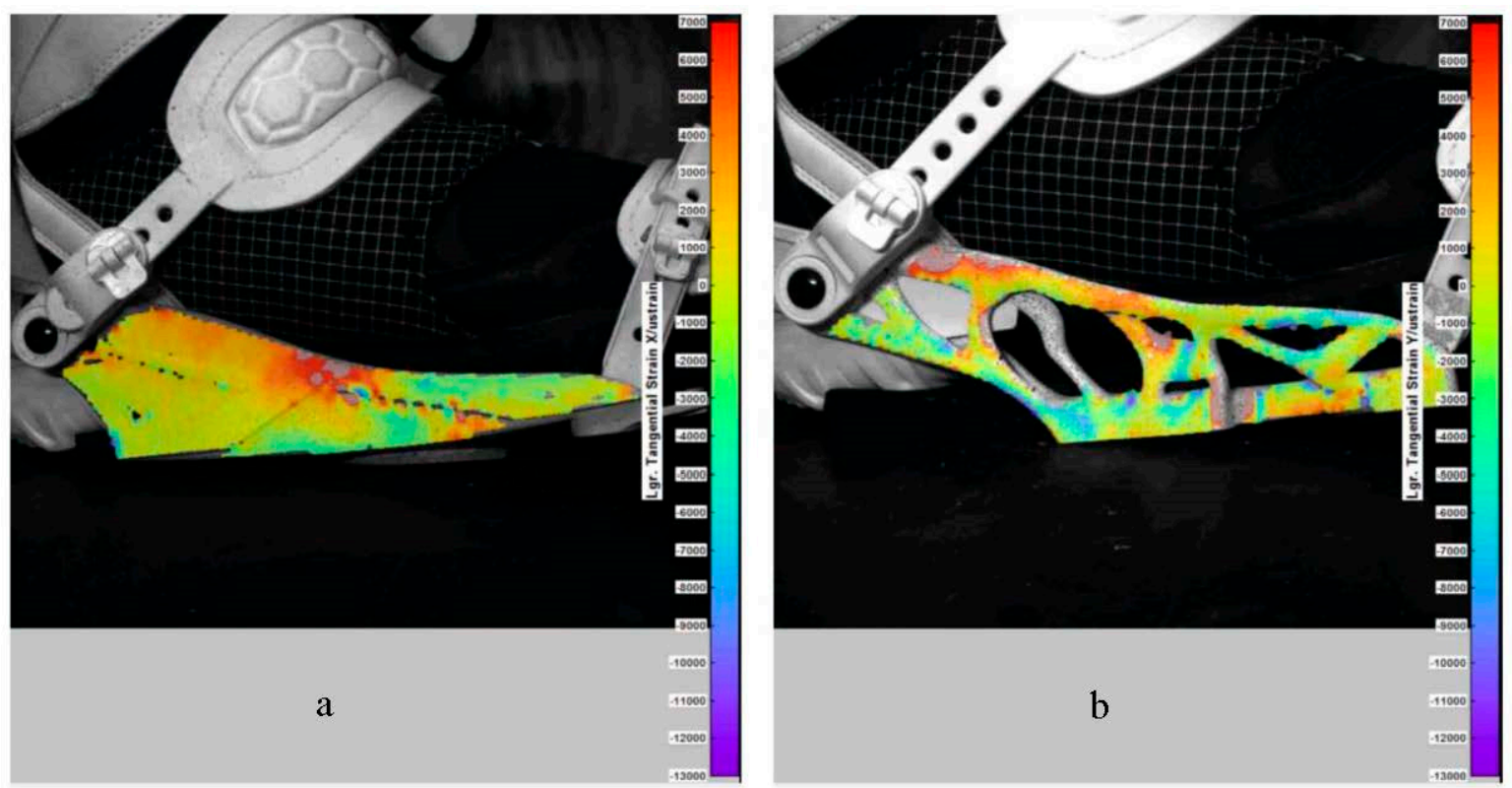

Figure 9. DIC comparison of deformations (horizonal axis) in plantarflexion of the commercial binding (a) and of the prototype (b).

\section{Conclusions}

The test conducted using the new procedure based on DIC and 3D printing shows that it is possible to develop rigid parts for sport equipment with reduced weight and improved performances. In particular, the DIC analysis of 3D-printed parts shows the same deformation patterns of injection-molded ones and allows one to determine the loads applied during the use, which can then be used for FEM simulation and topological optimization. Nevertheless, even without the use of FEM analysis, this approach allows one to determine the eventual points of stress concentration and the use of 3D printing, significantly reducing the time of every iteration necessary to obtain to the optimal weight/performance ratio. A statistical approach to precisely define the differences in all parts of different bindings will be performed in a following study. This approach can be also applied to other parts made of thermoplastic and elastomeric materials used in sport equipment (e.g., ski boots, rollerblades, etc.). 
Acknowledgments: The authors thanks Union bindings for providing the bindings and for the 3D printing.

Conflicts of Interest: The authors declare no conflict of interest.

\section{References}

1. Colonna, M.; Nicotra, M.; Moncalero, M. Materials, designs and standards used in ski-boots for alpine skiing. Sports 2013, 1, 78-113.

2. Petrone, N.; Marcolin, G.; Panizzolo, F.A. The effect of boot stiffness on field and laboratory flexural behavior of alpine ski boots. Sports Eng. 2013, 16, 265-280.

3. Kajtaz, M.; Subic, A. Three-Dimensional Printing of Sports Equipment. In Materials in Sports Equipment; Woodhead Publishing: Cambridge, UK, 2019; pp. 161-198.

4. Vasquez, M.; Haworth, B.; Hopkinson, N. Methods for quantifying the stable sintering region in laser sintered polyamide-12. Polym. Eng. Sci. 2013, 53, 1230-1240.

5. Collins, P.K.; Leen, R.; Usma Alvarez, C. User centric design, data analysis and performance of snowboard bindings. Proc. Eng. 2016, 147, 437-442.

(C) 2020 by the authors. Licensee MDPI, Basel, Switzerland. This article is an open access article distributed under the terms and conditions of the Creative Commons Attribution (CC BY) license (http://creativecommons.org/licenses/by/4.0/). 